\title{
Top-quark production at hadron colliders
}

\author{
Roberto Bonciani*† \\ Laboratoire de Physique Subatomique et de Cosmologie, Université Joseph \\ Fourier/CNRS-IN2P3/INPG, F-38026 Grenoble, France \\ E-mail: roberto.bonciani@lpsc.in2p3.fr

\section{Andrea Ferroglia} \\ New York City College of Technology 300 Jay Street, NY 11201 Brooklyn, US \\ E-mail: AFerroglia@citytech.cuny.edu
}

The current theoretical predictions for the observables related to the top-quark pair and the singletop productions at hadron colliders are briefly reviewed. The theoretical predictions are compared to the experimental measurements carried out at Tevatron and LHC.

XXIst International Europhysics Conference on High Energy Physics

21-27 July 2011

Grenoble, Rhône-Alpes France

\footnotetext{
*Speaker.

†Work partly supported by the initiative "Projets de Physique Théorique de l'IN2P3".

$¥$ Work supported by the PSC-CUNY Award N. 64133-00 42 and by the NSF grant PHY-1068317.
} 


\section{Introduction}

The top quark is the heaviest elementary particle produced so far at colliders. Due to its large mass, it is expected to interact strongly with the electroweak symmetry breaking sector of the Standard Model (SM). Consequently, the top-quark plays a key role in the investigation of the origin of particle masses, both in the SM and in models of "new physics" (NP).

At hadron colliders, top quarks are produced via two production mechanisms: $i$ ) in "pairs", $p p(\bar{p}) \rightarrow t \bar{t}$ and $i i)$ as "single tops", $p p(\bar{p}) \rightarrow t \bar{b}, p p(\bar{p}) \rightarrow t q(\bar{q})$, or $p p(\bar{p}) \rightarrow t W$. The pair production process occurs more than twice as often as the single top production. Moreover, its experimental signature is cleaner. For these reasons, the top quark was originally discovered in $t \bar{t}$ events and it took 14 more years to detect single-top production events at the Tevatron.

The top quark has a very short lifetime: it decays almost exclusively in a $b$ quark and a $W$ boson in $\sim 5 \cdot 10^{-25} \mathrm{~s}$. Since the top-quark lifetime is about one order of magnitude smaller than the hadronization time, the top is the only quark which does not hadronize. Consequently, the topquark quantum numbers are accessible to the experimental measurements. Its spin and the exact nature of its couplings to the $W$ boson can be studied starting from the geometrical distribution of the decay products. The top-quark mass, which together with the $W$ mass plays an important role in constraining the Higgs mass via radiative corrections, can be measured with great accuracy, provided that a satisfactory theoretical definition of this parameter is used.

The top pair and the single top production processes at hadron colliders can also be regarded as background for NP processes. In this short review the top quark events are considered as signal.

In the following we briefly review the status of the measurements of top-quark observables at Tevatron (for references see the CDF and D0 web pages [1,2]) and at the LHC (see the CMS and ATLAS web pages $[3,4]$ ) and we summarize the corresponding theoretical predictions.

\section{Top-Quark at the Tevatron}

From the top-quark discovery in 1995 and until the shutdown on September 30, 2011, the top-quark production and decay have been extensively studied at the Fermilab Tevatron.

The first top-related observable measured at the Tevatron was the total pair-production cross section, defined as $\sigma_{t \bar{t}}=\left(N-N_{b k g}\right) /(\varepsilon L)$, where $N$ is the number of measured events, $N_{b k g}$ is the number of background events, simulated by a MC event generator, $\varepsilon$ is the pair selection efficiency (also simulated with a $\mathrm{MC}$ ), and $L$ is the luminosity, measured with data-driven techniques. A recent measurement of $\sigma_{t \bar{t}}$ by the CDF collaboration gives $\sigma_{t \bar{t}}^{(C D F)}=7.5 \pm 0.48 \mathrm{pb}$. The experimental error corresponds to a relative error of only $\Delta \sigma_{t \bar{t}} / \sigma_{t \bar{t}} \sim 6.5 \%$. The measured pair production cross section is in good agreement with the SM value (See Section 4). For what concerns the single top production, the current combined Tevatron measurement of the $(s+t)$-channel cross section is $\sigma_{s+t}=2.76_{-0.47}^{+0.58} \mathrm{pb}$, which is in good agreement with the SM value. This measurement also allows one to determine $\left|V_{t b}\right|$. However, the CDF collaboration registers a tension in the ratio of the $s$ - and $t$-channel cross sections. The measured ratio is more than $2 \sigma$ away from the SM prediction.

The current Tevatron combination of top-quark mass measurements is $m_{t}=173.2 \pm 0.9 \mathrm{GeV}$, with a relative error of only $\sim 0.5 \%$. However, given the fact that the measurement is carried out by comparing data with MC simulations, and since the top-mass parameter used in the MC is not well 
defined theoretically, it would be desirable to have a measurement of $m_{t}$ related to a well defined Lagrangian parameter. Recently, the D0 collaboration evaluated the on-shell and $\overline{\mathrm{MS}}$ top masses by comparing data to the most up-to-dated theoretical predictions for the production cross section, finding a mean value for the on-shell $m_{t}$ which is slightly below the value of $173.2 \mathrm{GeV}$, but still compatible with it within one standard deviation. The width of the top quark is also measured at Tevatron. The limit reported by CDF is $\Gamma_{t}<7.6 \mathrm{GeV}$ at the $95 \% \mathrm{CL}$, and the value measured by D0 is $\Gamma_{t}=1.99_{-0.55}^{+0.69} \mathrm{GeV}$. The difference between the top and the anti-top masses was measured by the D0 collaboration and it is compatible with zero: $\Delta m_{t}=0.8 \pm 1.8$ (stat) \pm 0.5 (syst $) \mathrm{GeV}$.

The $W$ helicity fractions, $F_{0}, F_{R}$ and $F_{L}$, are measured fitting the one-parameter distribution of the positive charged lepton coming from the $W$ decay. Using CDF and D0 measurements that simultaneously determine $F_{0}$ and $F_{R}$, one finds $F_{0}=0.732 \pm 0.081, F_{R}=-0.039 \pm 0.045$, in full agreement with the NNLO SM predictions [5]. Also the spin correlations are in agreement with the SM predictions [6]. D0 measures a $t \bar{t}$ spin correlation strength, using as spin quantization axis the direction of the beam, of $C=0.1_{-0.45}^{+0.45}$, while CDF finds $C=0.72 \pm 0.64$ (stat) \pm 0.26 (syst).

Tevatron experiments are also searching for NP in top-quark pair and single-top production processes. However, so far, no evidence of NP was found, and the good agreement with the SM predictions is used to set constraints on the NP parameters, such as the masses and the couplings of the conjectured NP particles. The only observable which exhibits a sizable $(\sim 2-3 \sigma)$ discrepancy with the corresponding SM prediction is the top-pair forward-backward asymmetry, for which we refer the reader to the dedicated review in these proceedings [7].

\section{Top-Quark at the LHC}

Since the end of 2010, also CMS and ATLAS collaborations at the LHC are producing accurate measurements of the top properties. The most recent values are based on $\sim 1 \mathrm{fb}^{-1}$ of data, recorded between the end of 2010 and Summer 2011.

The $t \bar{t}$ production cross section was measured by both collaborations: the measured values are $\sigma_{t \bar{t}}^{(C M S)}=158 \pm 19 \mathrm{pb}$ and $\sigma_{t \bar{t}}^{(\text {ATLAS })}=176 \pm 5(\text { stat })_{-10}^{+13}($ syst $) \pm 7($ lum $) \mathrm{pb}$. Therefore, after only few months of data taking, the relative error on this observable is already quite small $(\sim 10-15 \%)$; furthermore the statistical uncertainty is already smaller than the systematic one. The t-channel single top production cross section is measured with a larger relative error of $\sim 30 \%: \sigma_{t}^{(C M S)}=$ $83.6 \pm 29.8($ stat + syst $) \pm 3.3($ lum $) \mathrm{pb}$ and $\sigma_{t}^{(A T L A S)}=90_{-22}^{+32} \mathrm{pb}$.

The mass of the top quark was measured by the ATLAS collaboration using a template method (which suffers of the same problems already pointed out in the previous section): the value obtained is $m_{t}^{(A T L A S)}=175.9 \pm 0.9$ (stat.) \pm 2.7 (syst. $) \mathrm{GeV}$. The CMS collaboration repeated the analysis done by D0, using the theoretical cross section and measuring the on-shell and $\overline{\mathrm{MS}}$ top masses, finding comparable results. The difference between the top and anti-top masses was measured by CMS, finding a value compatible with zero: $\Delta m_{t}=-1.2 \pm 1.2$ (stat. $) \pm 0.5$ (syst.) $\mathrm{GeV}$.

$W$-helicity fractions and spin correlations, measured by ATLAS, are also compatible with their SM value: $F_{0}=0.75 \pm 0.08, F_{L}=0.25 \pm 0.08$ (setting $F_{R}=0$ ), and $\kappa=0.34_{-0.11}^{+0.15}$ in the helicity base.

The already remarkable accuracy of the LHC measurements is going to improve in the next years. For example, in the high-luminosity and high-energy phase, the $t \bar{t}$ production cross section 
is expected to be measured with an accuracy of 5\%, while the single-top $t$-channel cross section will be measured with an accuracy of $10 \%$. These very precise experimental measurements must be matched by equally accurate theoretical predictions.

\section{Theoretical Predictions}

The production of a top-antitop pairs is dominated by the strong interaction. The inclusive production cross section can be written using the QCD Factorization Theorem as

$$
\sigma_{h_{1}, h_{2}}^{i \bar{t}}\left(s_{\text {had }}, m_{t}^{2}\right)=\sum_{i j} \int_{4 m_{t}^{2}}^{s_{\text {had }}} d \hat{s} L_{i j}\left(\hat{s}, s_{\text {had }}, \mu_{f}^{2}\right) \hat{\sigma}_{i j}\left(\hat{s}, m_{t}^{2}, \mu_{f}^{2}, \mu_{r}^{2}\right)
$$

where the hard scattering of the partons $i$ and $j(i, j \in\{q, \bar{q}, g\})$ at a partonic center of mass energy $\hat{s}$ is described by the partonic cross section, $\hat{\sigma}_{i j}$, which can be calculated in perturbative QCD. The process independent partonic luminosity, $L_{i j}$, describes the probability of finding, in the hadrons $h_{1}$ and $h_{2}\left(h_{1}, h_{2}=p, \bar{p}\right.$ at the Tevatron, $h_{1}, h_{2}=p, p$ at the LHC), an initial state involving partons $i$ and $j$ with the given partonic energy $\hat{s}$. The integration extends up to the collider hadronic c. $\mathrm{m}$. energy $s_{\text {had }} . \mu_{f}$ and $\mu_{r}$ indicate the renormalization and factorization scales.

At LO in perturbation theory, there are two partonic channels contributing to the $t \bar{t}$ production cross section: the quark-antiquark channel $q \bar{q} \rightarrow t \bar{t}$ and the gluon fusion channel $g g \rightarrow t \bar{t}$. Because of the interplay between parton luminosity and partonic cross sections, the quark-antiquark channel dominates at the Tevatron, while at the LHC the inclusive cross section is largely dominated by gluon fusion events.

In single-top production, there are three LO partonic channels: i) $q(\bar{q}) b \rightarrow q^{\prime}\left(\bar{q}^{\prime}\right) t$, in which a $W$ boson is exchanged in the $t$ channel, ii) $q \bar{q}^{\prime} \rightarrow t \bar{b}$, in which the $W$ boson is exchanged in the $s$ channel, and iii) the "associated $t W$ production" $g b \rightarrow t W$. The $t$-channel process dominates the single top production both at the Tevatron and at the LHC. The $s$-channel production was detected at the Tevatron and it plays no role at the LHC. The associated production, instead, cannot be revealed at the Tevatron while is the second most important single-top production mechanism at the LHC.

\subsection{The NLO Calculations}

The LO predictions for the $t \bar{t}$ or single-top production cross sections are affected by a huge dependence on the renormalization/factorization scales, and they cannot be regarded as reliable predictions. More accurate predictions can be obtained by taking into account the NLO corrections, which consist of two parts: the virtual corrections, originating from the interference of the oneloop diagrams with the tree-level ones, and the real radiation corrections, originating from the interference of the $2 \rightarrow 3$ amplitudes. For totally inclusive quantities, as for instance the total cross section, one has to integrate the final-state particles over the complete phase space. This is the approach used for instance in [8]. In so doing, the IR divergences of the virtual part cancel exactly (analytically) against the divergences that come from the integration of the extra parton in particular regions of the phase space. For a comparison with the experimental measurements, however, one needs to impose cuts and to take into account the geometrical acceptance of the detectors. For more exclusive observables, therefore, a subtraction scheme is needed to "regularize" the IR collinear and 
soft divergences, coming from the integration over the phase space. At the NLO, the subtraction terms are completely known and several subtraction formalisms are available in the literature [9].

Let us first consider the $t$ and $\bar{t}$ in the final state as stable on-shell particles. The NLO QCD corrections to the total $t \bar{t}$ cross section, summed over the final spins and colors, were calculated by many groups [10]. They enhance the cross section by almost $25 \%$ at Tevatron and by $50 \%$ at the LHC. The residual renormalization/factorization scale dependence, plus parton distribution functions uncertainties, is about $15-20 \%$. The EW corrections are also known [11], but their contribution ( $+1 \%$ at Tevatron and $-0.5 \%$ at the $\mathrm{LHC})$ is negligible in comparison to the QCD theoretical error.

The NLO QCD corrections to the $t$-channel single-top production are moderate. They enhance the cross section by $9 \%$ at the Tevatron and by $5 \%$ at the LHC $[12,13]$. The NLO EW corrections decrease the cross section by $1 \%$ both at the Tevatron and the LHC [14]. The NLO QCD corrections to the $s$-channel cross section are large, resulting in an enhancement of $47 \%$ at the Tevatron and $44 \%$ at the LHC $[13,15]$. Finally, the NLO QCD corrections to the associated $t W$ production enhance the cross section by $10 \%$ at the LHC [16].

For what concerns processes with additional particles in the final state, the NLO corrections to $t \bar{t}+j$ were calculated in [17] (the calculated cross section at the Tevatron, $\sigma_{t \bar{t} j}=1.79_{-0.31}^{+0.16} \mathrm{pb}$ is in good agreement with the CDF mesurement) and those to $t \bar{t}+2 j$ in [18]. Moreover, the $t \bar{t} b \bar{b}$ production was considered in [19]. The NLO corrections to the production of a top pair in association with a photon were calculated in [20].

The QCD corrections to processes that involve at least two large energy scales (the partonic energy $\sqrt{\hat{s}}$ and the top mass $m_{t}$, are both much larger than $\Lambda_{Q C D}$ ) are characterized by a logarithmic behavior in the vicinity of the boundary of the phase space. The logarithmically enhanced terms spoil the convergence of the fixed-order expansion, and a systematic resummation of the logarithms to all orders in perturbation theory becomes necessary. A comprehensive review of the results obtained with resummation techniques in top-quark physics can be found in [21].

In the works mentioned up to this point, the top quarks are treated as stable particles. However, only hadrons and leptons are detected experimentally and it is on these particles that the experimental cuts are imposed. Therefore, it is highly desirable to consider the actual final state in the theoretical analyses. This is very difficult from the point of view of the calculation, since one needs to deal with Feynman diagrams with many external legs. A first step towards this goal consists in working within the "narrow-width approximation": since the top-quark behaves as a narrow resonance, i.e. $\Gamma_{t} / m_{t} \ll 1$, one can formally take the limit $\Gamma_{t} / m_{t} \rightarrow 0$ of the complete cross section. The limit $\Gamma_{t} / m_{t} \rightarrow 0$ decouples the top-quark production process from the top-quark decay. This approach allows to compute realistic distributions and to keep trace of the spin of the tops. Two groups performed detailed studies of the top-quark pair production within the narrow width approximation approach [6, 22, 23]. For single-top production, the same formalism was used to study the $t$-channel cross section at the LHC [24]. Recently, also the off-shell effects both for $t$ and $s$-channel cross sections at Tevatron and at the LHC were computed [25].

In 2010, two groups calculated the complete set of corrections to $p p(\bar{p}) \rightarrow W^{+} W^{-} b \bar{b}$, including also the non-factorizable corrections [26]. As a by-product of the calculation, the authors could prove that for inclusive quantities the non-factorizable corrections are indeed of $\mathscr{O}\left(\Gamma_{t} / m_{t}\right) \sim 1 \%$. With these results many exclusive observables, with realistic experimental cuts, can be evaluated. 


\subsection{Outlook: Towards a Complete NNLO Analysis of the Top-Pair Production in Perturbative QCD}

The foreseen accuracy with which LHC will be able to measure some of the top-pair production observables is such that in several cases the calculation of the NNLO corrections is required. Due to the complexity of the calculations, to date the complete set of NNLO QCD corrections is not yet available. However, many partial results are known, and the full calculation of the NNLO correction appears to be within reach. The most accurate theoretical predictions currently used for comparison with the experimental measurements include the "approximate NNLO" corrections [27] (NLO plus some or all of the following ingredients: scale dependence at NNLO, Coulomb terms up to two loops, logarithmic terms obtained by re-expanding NNLL formulas).

Many parts of the full top-pair NNLO matrix element are known. In [28] the matrix elements for the $q \bar{q}$ and $g g$ channels were computed in the $s \gg m_{t}^{2}$ limit. In [29], matrix elements in the $q \bar{q}$ channel were computed numerically and by retaining the full dependence on the top-quark mass. In [30], all of the IR two-loop poles, both in the $q \bar{q}$ and $g g$ channels, were evaluated analytically. In [31], the virtual one-loop times one-loop matrix elements were calculated. Finally, in [32] the two-loop fermionic and leading color corrections to the $q \bar{q}$ channel and the two-loop leading

color corrections to the $g g$ channel were analytically computed by using the Laporta algorithm (implemented in the computer code Reduze [33]) and the differential equations method (see [35]). A subtraction scheme for the real corrections at the NNLO is developed in [34].

\section{References}

[1] http://www-cdf.fnal.gov/physics/new/top/public_xsection.html

[2] http://www-d0.fnal.gov/Run2Physics/top/index.html

[3] https://twiki.cern.ch/twiki/bin/view/CMSPublic/PhysicsResultsTOP

[4] https://twiki.cern.ch/twiki/bin/view/AtlasPublic/TopPublicResults

[5] A. Czarnecki, et al., Phys. Rev. D81, 111503 (2010).

[6] W. Bernreuther, et al., Phys. Rev. Lett. 87, 242002 (2001). Nucl. Phys. B690, 81-137 (2004).

[7] S. Westhoff, arXiv:1108.3341 [hep-ph].

[8] M. Czakon, A. Mitov, Nucl. Phys. B824, 111-135 (2010).

[9] S. Catani, M. H. Seymour, Nucl. Phys. B485 291-419 (1997). S. Catani, et al., Nucl. Phys. B627, 189-265 (2002). L. Phaf, S. Weinzierl, JHEP 0104006 (2001). S. Frixione, et al., Nucl. Phys. B467, 399-442 (1996). Z. Nagy, Z. Trocsanyi, Nucl. Phys. B486, 189-226 (1997). C. H. Chung, et al., JHEP 1106, 144 (2011). A. Gehrmann-De Ridder, M. Ritzmann, JHEP 0907, 041 (2009).

[10] P. Nason et al. Nucl. Phys. B 303607 (1988); Nucl. Phys. B 327 49(1989) [Erratum-ibid. B 335260 (1990)]. W. Beenakker et al. Phys. Rev. D 4054 (1989). W. Beenakker et al. Nucl. Phys. B 351507 (1991). M. L. Mangano et al. Nucl. Phys. B 373, 295 (1992).

[11] W. Beenakker et al. Nucl. Phys. B 411, 343 (1994). W. Bernreuther et al. Phys. Lett. B 633, 54 (2006); Phys. Rev. D 74, 113005 (2006).

[12] G. Bordes, B. van Eijk, Nucl. Phys. B 435, 23 (1995). T. Stelzer, et al., Phys. Rev. D 56, 5919 (1997). Phys. Rev. D 58, 094021 (1998). 
[13] B. W. Harris, et al., Phys. Rev. D 66054024 (2002).

[14] M. Beccaria, et al., Phys. Rev. D 74, 013008 (2006). M. Beccaria, et al., Phys. Rev. D 77, 113018 (2008).

[15] M. C. Smith, S. Willenbrock, Phys. Rev. D 54, 6696 (1996). Z. Sullivan, Phys. Rev. D70, 114012 (2004), Phys. Rev. D72, 094034 (2005).

[16] W. T. Giele, et al., Phys. Lett. B 372, 141 (1996). S. Zhu, Phys. Lett. B 524, 283 (2002) [Erratum-ibid. B 537, 351 (2002)].

[17] S. Dittmaier, et al., Eur. Phys. J. C 59625 (2009), Phys. Rev. Lett. 98262002 (2007). K. Melnikov, M. Schulze, Nucl. Phys. B 840129 (2010).

[18] G. Bevilacqua, et al., Phys. Rev. Lett. 104, 162002 (2010).

[19] A. Bredenstein, et al., JHEP 0808, 108 (2008), Phys. Rev. Lett. 103, 012002 (2009), JHEP 1003, 021 (2010).

[20] K. Melnikov, et al., Phys. Rev. D 83074013 (2011).

[21] N. Kidonakis, B. D. Pecjak, arXiv:1108.6063 [hep-ph].

[22] W. Bernreuther, Z. -G. Si, Nucl. Phys. B837, 90-121 (2010).

[23] K. Melnikov, M. Schulze, JHEP 0908, 049 (2009).

[24] R. Schwienhorst, et al., Phys. Rev. D83, 034019 (2011).

[25] P. Falgari, et al., Phys. Rev. D83, 094013 (2011).

[26] A. Denner, et al., Phys. Rev. Lett. 106, 052001 (2011). G. Bevilacqua, et al., JHEP 1102, 083 (2011).

[27] S. Moch and P. Uwer, Phys. Rev. D 78034003 (2008). N. Kidonakis and R. Vogt, Phys. Rev. D 78 074005 (2008). U. Langenfeld et al. Phys. Rev. D80, 054009 (2009). M. Aliev, et al., Comput. Phys. Commun. 182, 1034-1046 (2011). V. Ahrens, et al. Phys. Lett. B 703135 (2011). M. Beneke, et al. arXiv:1109.1536 [hep-ph].

[28] M. Czakon et al. Phys. Lett. B 651147 (2007), Nucl. Phys. B 798210 (2008).

[29] M. Czakon, Phys. Lett. B 664307 (2008).

[30] A. Ferroglia, et al., Phys. Rev. Lett. 103201601 (2009), JHEP 0911062 (2009).

[31] J.G. Körner, et al., Phys. Rev. D 73034030 (2006) . Phys. Rev. D 77094011 (2008) . C. Anastasiou, S. M. Aybat, Phys. Rev. D 78114006 (2008) . B. Kniehl, et al., Phys. Rev. D 78094013 (2008) .

[32] R. Bonciani, et al., JHEP 0807129 (2008), [arXiv:0810.0598 [hep-ph]]. R. Bonciani, et al., JHEP 0908067 (2009). R. Bonciani, et al., JHEP 1101, 102 (2011), PoS ICHEP2010, 098 (2010).

[33] C. Studerus, Comput. Phys. Commun. 1811293 (2010). A. von Manteuffel, C. Studerus, Reduze 2, to be published.

[34] D.A. Kosower, Phys. Rev. D 67116003 (2003). A. Daleo, et al., JHEP 0704016 (2007). A. Gehrmann-De Ridder, et al., JHEP 0509056 (2005). E. W. Nigel Glover, J. Pires, JHEP 1006096 (2010). R. Boughezal, et al., JHEP 1102, 098 (2011). S. Catani, M. Grazzini, Phys. Rev. Lett. 98 222002 (2007). M. Czakon, Phys. Lett. B693, 259-268 (2010). Nucl. Phys. B849, 250-295 (2011). I. Bierenbaum, et al., arXiv:1107.4384 [hep-ph]. C. Anastasiou, et al., JHEP 1103, 038 (2011).

[35] A. von Manteuffel, these proceedings. 